\title{
Snake Classifier: Aplicativo mobile para classificação de serpentes peçonhentas
}

\author{
Emanuel A. Machiaveli ${ }^{1}$, Juan Morysson Viana Marciano ${ }^{1}$, Felipe Gonçalves dos \\ Santos ${ }^{1}$
}

${ }^{1}$ Instituto Federal de Educação, Ciência e Tecnologia do Piauí - IFPI - Campus

Corrente

emanuelmachiavelli@gmail.com, juan.morysson@ifpi.edu.br, felipe.santosdifpi.edu.br

\begin{abstract}
Snakes are at the top of the list of accidents with venomous animals in Brazil. The little knowledge of the population about these animals, makes their identification difficult in cases of accidents, when it is necessary for the application of the correct antiophidic serum. It was proposed in this work, with the use of Artificial intelligence with the application of Transfer Learning techniques, the creation of a model of neural network, implemented to a cell phone application, where the species classification is done instantly. The model trained with a bank of 3401 images obtained an accuracy level of $90.22 \%$. The tests carried out with the application in operation, on the other hand, led to a hit level of $88.41 \%$ after testing it with 164 images.
\end{abstract}

Keywords: Snakes. Neural network. Transfer Learning. Application.

Resumo. Serpentes estão no topo da lista de acidentes com animais peçonhentos no Brasil. O pouco conhecimento da população a respeito desses animais, torna sua identificação dificultada em casos de acidentes, quando se faz necessário a classificação para a aplicação do soro antiofídico correto. Foi proposto neste trabalho, com o uso de Inteligência Artificial com aplicação das técnicas de Transfer Learning, a criação de um modelo de rede neural, implementado a um aplicativo de celular onde, a classificação da espécie é feita de forma instantânea. O modelo treinado com um banco de 3401 imagens obteve um nível acurácia de $90.22 \%$. Já os testes realizados com o aplicativo em funcionamento, levou a uma taxa de acerto de $88.41 \%$ após testá-lo com 164 imagens.

Palavras chaves: Serpentes. Redes Neurais. Transfer Learning. Aplicativo.

\section{Introdução}

De acordo com o Ministério da Saúde, no Brasil, no ano de 2017 ocorreram cerca de 28 mil acidentes ofídicos. No caso das serpentes, o acidente ofídico é o quadro de envenenamento que ocorre através da inoculação do veneno por meio das glândulas venenosas presentes nas presas do animal. Podendo ser de forma intravenosa ou até mesmo quando em contato com os olhos do indivíduo.

Segundo o próprio ministério, através de dados coletados durante um período de dez anos, entre 2007 e 2017, serpentes são os animais peçonhentos que mais matam no país, seguidos de ataques de escorpião e abelhas. No total de 2792 mortes registradas, 1271 casos ou $45 \%$ do total foram ocasionados por ataques de cobras venenosas. 
Dentre as 412 espécies catalogadas de cobras existentes no país, somente quatro tipos são responsáveis por esses números como animais com potencial de levar risco a vida dos seres humanos, somente, serpentes do gênero Bothrops (Jararaca e suas subespécies), Crotalus (Cascavel), Lachesis (exclusivamente surucucu-pico-de-jaca) e Micrurus e LeptoMicrurus (Coral Verdadeira).

Um dos procedimentos recomendados em caso de acidente, é o transporte do animal vivo ou morto até o local de atendimento da vítima para a identificação da espécie, a fim de fornecer as medidas profiláticas corretas com a aplicação do soro antiofídico indicado no combate à ação da toxina injetada pelo animal.

No entanto, pode ser uma medida perigosa, já que se trata da manipulação de animais silvestres, por parte de indivíduos não capacitados, que pode até mesmo acabar gerando novos acidentes.

Visto que se faz necessário a disponibilização de um mecanismo capaz de fazer a distinção das espécies, foi proposto neste trabalho, a criação de uma ferramenta que possibilita a classificação de serpentes peçonhentas por meio de imagens utilizando inteligência artificial, mais precisamente na subárea de reconhecimento de imagens fazendo o uso de redes neurais convolucionais.

O mesmo tem a finalidade de ajudar nesse processo de identificação em caso de contato de pessoas com esses animais ou em casos de acidentes para possibilitar a identificação do gênero do animal para facilitar o atendimento a vítima e aplicação do medicamento correto para cada caso, a ferramenta pode também desenvolver ou aprimorar o conhecimento científico do usuário do aplicativo acerca das espécies da fauna brasileira.

O presente trabalho tem como objetivo fazer a classificação e diferenciação entre cobras venenosas e não venenosas por meio de um aplicativo para dispositivos móveis, utilizando técnicas de aprendizagem por transferência. Que vai possibilitar fazer a identificação do gênero do animal em casos de acidente, além de despertar no usuário o interesse pela ciência e preservação ambiental, apresentando as informações e principais características de cada espécie classificada.

\section{Trabalhos relacionados}

Para a realização deste projeto, foi feita a revisão literária de trabalhos relacionados na mesma temática, onde são de suma importância para a criação de uma base sólida de conhecimento específico na área de desenvolvimento.

Marques (2019), no seu projeto, desenvolveu um modelo de aprendizado profundo empregado no objetivo de identificar e classificar 5 espécies de plantas daninhas que aparecem como pragas dando prejuízos nas lavouras, principalmente as mais resistentes aos herbicidas comerciais.

A intenção foi aplicar e comparar a performance de quatro arquiteturas de redes neurais convolucionais para a classificação de plantas daninhas de cinco espécies contidas em um banco de imagens. Tendo como propósito ajudar o agricultor a encontrar a planta indesejada, para fazer sua eliminação da forma mais adequada.

Trabalho que se assemelha em termos de avaliação de classificadores de aprendizagem de máquina com Britto (2020) que determina e qualifica a precisão de 
algoritmos aplicados na classificação de segmentos de imagens, empregados no desenvolvimento de um sistema de identificação de plantas medicinais por meio de características de cores, formas e texturas apresentadas nas imagens.

Em seu trabalho Nardelli (2020), propôs a criação de um aplicativo de celular, utilizando sistema de redes neurais com TensorFlow para classificação de imagens, com a finalidade de desenvolver uma ferramenta para auxiliar no reconhecimento das principais espécies de camarões comercializadas no estado de Santa Catarina. A principal funcionalidade do seu trabalho é classificar qual a espécie de camarão identificada, assim como o percentual de acerto feito pelo algoritmo de classificação. Fazendo o uso da própria câmera do aparelho dentro do aplicativo.

\section{Desenvolvimento.}

Este presente trabalho foi dividido em duas etapas principais, a primeira delas foi a criação do banco de imagens para realizar treinamento dos dados para o modelo personalizado de Machine learning a ser usado na identificação e implantação no aplicativo. Depois foi realizado o processo de desenvolvimento da própria aplicação mobile, a qual utiliza do modelo já previamente treinado para a amostragem dos resultados relacionados a detecção de classificação das espécies.

\subsection{Redes Neurais}

As Redes Neurais Artificiais (RNAs) são ferramentas de Inteligência Artificial que possuem a capacidade de se adaptar e de aprender a realizar certa tarefa, ou simular ações do comportamento humano, a partir de um conjunto de exemplos dados como base (OSÓRIO, 2000). Por meio das redes neurais projetam-se máquinas que são capazes de exibir um comportamento inteligente, como se fossem reações humanas.

Transfer Learning ou aprendizado por transferência é uma área de pesquisa em machine learning que concentra em armazenar o conhecimento adquirido ao resolver um problema e aplicá-lo em um problema diferente, porém relacionado.

Esse método de aprendizagem de máquina, torna-se viável em decorrência da dificuldade de se obter um conjunto de dados grande o suficiente para o treinamento de Redes Neurais Convolucionais (CNNs) do zero. Tendo em vista a utilização de um banco de dados pequeno para treinamento de modelos em Deep Learning, a utilização de aprendizado por transferência passa a ser a forma mais eficiente para alcançar bons resultados.

Como as redes construídas em Deep Learning são muito grandes, exigem um poder também muito grande de máquinas para fazer o processamento dos dados e treinamento dessas redes. Na técnica de Transfer Learning, é retirada dessa rede neural pré treinada, camadas posteriores desnecessárias onde é feito o congelamento dessas camadas, então é preservada uma parte do conhecimento dessa rede e depois aplicadas as novas camadas customizadas a essa arquitetura para resolução do problema específico.

\subsection{Banco de Imagens}

A princípio, a obtenção das imagens para criação de um banco para efetuar o treinamento na rede neural, se deu através da busca no Google imagens, por meio da pesquisa pelo nome científico para a obtenção de resultados referentes às 4 espécies distintas. Foi 
utilizada também a extensão do Google, Batch Image Download que permite baixar as imagens em lote. Para esse treinamento inicial foi formado um banco com 1328 imagens e posteriormente o número foi aumentado para 2401 imagens.

Para que fosse possível a criação dos rótulos das serpentes, foi necessário aprender as principais características de cada um dos gêneros de forma manual, onde algumas fontes serviram para aprimorar o conhecimento nessa área da biologia, como algumas fontes literárias, como a demonstrada na figura 1:

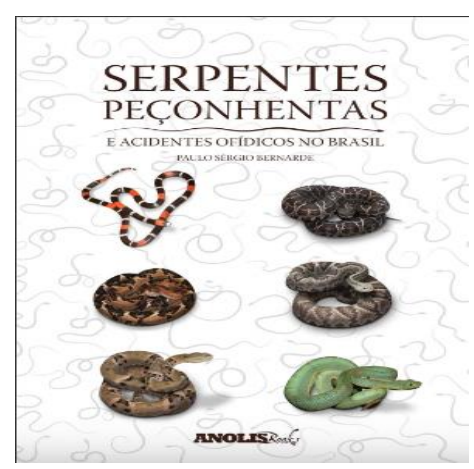

Figura 1. Serpentes peçonhentas e herpetologia no Brasil

Para a realização do treinamento das imagens, foi dividido e organizado 4 rótulos separados por pastas de acordo com as espécies expostas anteriormente como peçonhentas, e um rótulo com imagens de serpentes não peçonhentas.

\begin{tabular}{|c|c|}
\hline Crotalus(Cascavel) & \\
\hline Micrurus (Coral Verdadeira) & \\
\hline Bothrops(Jararacas) & \\
\hline Lachesis (Surucucu-pico-de-jaca) & \\
\hline Serpentes sem peçonha & \\
\hline
\end{tabular}

Figura 2. Gêneros das serpentes utilizadas como rótulos. 
As imagens capturadas foram armazenadas no Google Drive, com uma divisão por pastas de $80 \%$ para teste, e $20 \%$ para validação do modelo como apresenta a figura 3.

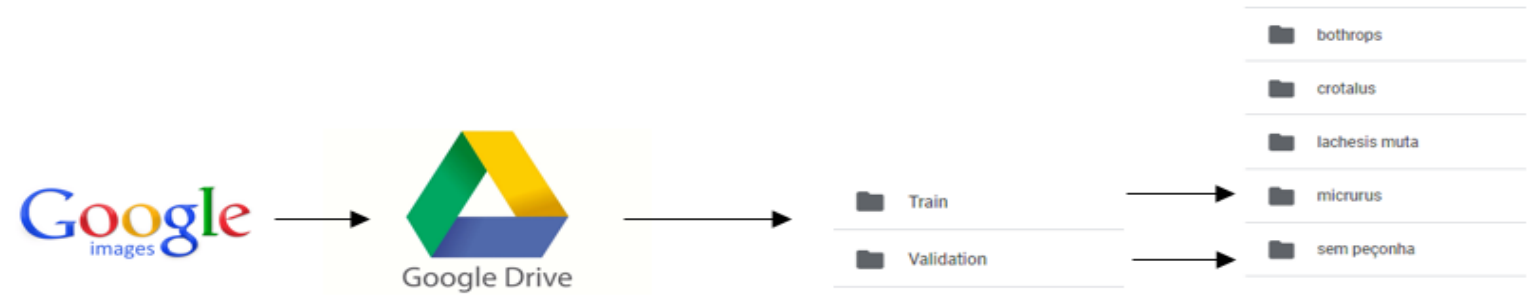

Figura 3. Separação dos rótulos por pastas.

Foi utilizado como ambiente para o desenvolvimento, Google Colab para execução de códigos python na nuvem. O qual possibilita, o uso de uma máquina virtual durante o processo de treinamento para melhorar o desempenho e diminuir o tempo de treinamento através do uso de uma GPU configurada neste ambiente na nuvem com 12.72GB de memória RAM e HD de 107.77 GB.

\subsection{MobileNetV2}

A arquitetura de rede neural utilizada para o método de transferência de aprendizagem foi a MobileNetV2, que é uma arquitetura de rede neural convolucional que busca um bom desempenho em dispositivos móveis (SANDLER et al., 2018). Focando na precisão quando se trata de recursos limitados, como é o caso de dispositivos móveis e embarcados. Diminuindo de forma significativa o número de operações e redução da memória necessária, porém mantendo a precisão.

Essa rede é previamente treinada na qual utiliza os pesos da ImageNet, um conjunto de dados de treinamento com 1,4 milhões de imagens de 1000 classes de objetos distintos. Utilizou-se do conhecimento dessa rede no processo de transfer learning, preservando sua arquitetura e seu conhecimento pré treinado e adicionando uma camada densa personalizada ao final dessa rede.

Foi instanciado para dentro do projeto em Python, O modelo MobileNetV2 pré carregado com os pesos da ImageNet mostrado na figura 4, sem incluir as camadas de classificação no topo (já que não possui muita utilidade), essa camada de topo representa a última camada seguindo a arquitetura do modelo de rede invertida, que vai de baixo pra cima.

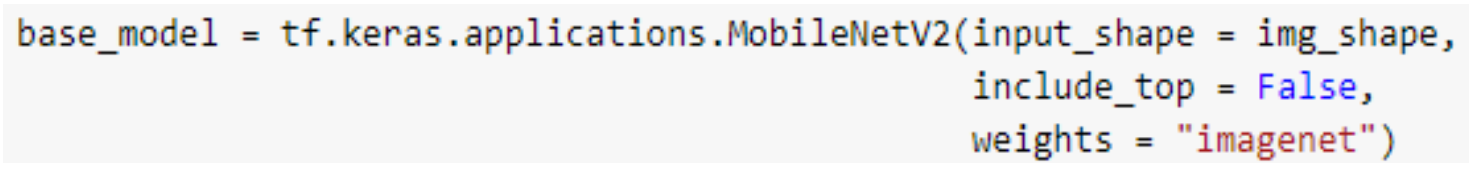

Figura 4 - download do modelo básico MobileNet V2.

Então é mais relevante preservar a camada anterior da operação de nivelamento para extração de recursos. Onde essa camada de gargalo, conserva mais generalidade quando comparado com a camada final que foi retirada. Outro fator importante antes de compilar e iniciar treinamento do modelo, é o congelamento da base convolucional. Isso previne que os pesos de uma determinada camada sejam usados no treinamento.

\subsection{TensorFlow}


Para o processo de aprendizagem de máquina, foi utilizado a linguagem python e a biblioteca TensorFlow, que segundo GINÊS(2018), é uma biblioteca de código aberto criada pelo Google para computação numérica e aprendizado de máquina em grande escala. A mesma fornece recursos para implementação em diversos casos como detecção de objetos, classificação de imagens e processamento de linguagem natural.

Após o treinamento e avaliação do modelo de transferência de aprendizagem, para salvar o modelo e torná-lo compatível para implementação em apps mobile, foi convertido para TensorFlow Lite, Biblioteca específica para implantação em dispositivos móveis e IOT. A mesma tem o intuito de possibilitar a otimização do modelo do TensorFlow para deixá-lo mais leve e rápido, criando assim um modelo compactado, para fazer a inferência e instalação no aplicativo do celular. A figura 5 mostra de forma resumida o ciclo de treinamento feito com TensorFlow.

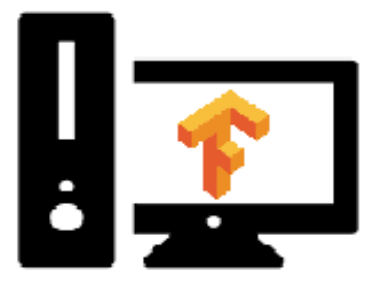

Treinamento do modelo

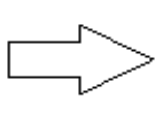

TensorFlow Lite

$$
\begin{aligned}
& \text { Otimização } \\
& \text { do modelo } \\
& \text { com TF Lite }
\end{aligned}
$$

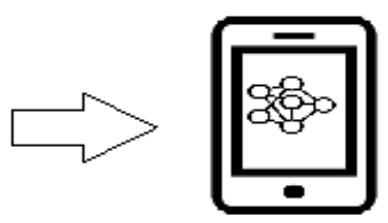

Implantação do modelo no dispositivo

Figura 5- Ciclo de treinamento do TensorFlow.

\subsection{Flutter}

A realização do aplicativo Snake Classifier foi feita com a utilização do framework Flutter. De acordo com DEVMEDIA (2019) Flutter é um framework construído pelo Google para facilitar o desenvolvimento de aplicativos multiplataforma (tanto para Android como IOS).

O Flutter surge com uma proposta que se assemelha com a ferramenta do Facebook para a criação de Apps híbridos, React Native, a qual utiliza Javascript na produção de aplicações de forma nativa utilizando um único código fonte.

Contudo, o Flutter vem se mostrando como ótima alternativa aos desenvolvedores de Apps móveis, muito por sua fluidez e alta performance.

O framework utiliza a linguagem de programação Dart, desenvolvida pelo Google em 2011, inicialmente focada para o desenvolvimento web, como alternativa para substituição do Javascript. A linguagem é orientada a objetos e sintaxe baseada em linguagem $\mathrm{C}$.

\section{Resultados}

Em uma primeira etapa de treinamento a arquitetura do MobilenetV2 teve todas suas camadas e parâmetros treináveis congelados onde os pesos da rede pré-treinada não foram atualizados durante o treinamento e foi adicionado uma camada densa ao final da rede com 5 neurônios e definindo 25 épocas de treinamento com essa camada personalizada.

$\mathrm{Na}$ avaliação para implantação do modelo, inicialmente foram utilizadas 1328 imagens para os 5 rótulos distintos para treinamento e validação do modelo. Em um 
primeiro teste com o modelo já treinado, foram utilizadas 164 imagens para teste no aplicativo, onde desse total, 139 imagens foram classificadas de forma assertiva dando um total de $84.75 \%$ de precisão do modelo. A tabela 1 mostra os dados respectivos a cada um dos rótulos e seu nível de precisão.

\begin{tabular}{|c|c|c|c|c|}
\hline Gênero/Espécie & $\begin{array}{c}\text { Total de } \\
\text { imagens } \\
\text { testadas }\end{array}$ & $\begin{array}{c}\text { Número de } \\
\text { acertos }\end{array}$ & $\begin{array}{c}\text { Número de } \\
\text { erros }\end{array}$ & $\begin{array}{c}\text { Porcentagem média } \\
\text { de acerto }\end{array}$ \\
\hline Crotalus (Cascavel) & 20 & 15 & 5 & $75 \%$ \\
\hline Bothrops (Jararaca) & 36 & 29 & 6 & $80,55 \%$ \\
\hline $\begin{array}{c}\text { Lachesis (Surucucu pico } \\
\text { de Jaca) }\end{array}$ & 36 & 30 & 6 & $83,33 \%$ \\
\hline Micrurus (Coral) & 32 & 30 & 2 & $93,75 \%$ \\
\hline Serpentes sem Veneno & 40 & 35 & 5 & $87,5 \%$ \\
\hline Total & 164 & 139 & 25 & $\mathbf{8 4 , 7 5 \%}$ \\
\hline
\end{tabular}

Tabela 1- análise da taxa de acerto do modelo no primeiro teste.

Ao analisar os resultados da tabela, é visto que o rótulo que apresentou a melhor taxa de acerto foi do gênero Micrurus (coral verdadeira) onde, dentre as 32 imagens utilizadas para teste no aplicativo 30 foram identificadas com êxito apresentando um total de 93,75\% na taxa de acerto. Seguindo depois o rótulo com imagens de serpentes sem veneno, destacando que esse rótulo possui uma variedade de espécies diferentes. Analisando os demais resultados, referente ao gênero Lachesis, o teste com o aplicativo Snake Classifier utilizando um total de 36 imagens obteve taxa de acerto de 83,33\%.

É possível analisar que as duas espécies que mostraram menor precisão de acerto no teste com o aplicativo foram os gêneros Crotalus com $75 \%$ contendo 5 erros do total de 20 imagens analisadas, onde esses falsos positivos eram na verdade do gênero Bothrops. Que aponta a semelhança das características entre ambos, como por exemplo sua coloração próxima. Também pôde ser observado que as imagens dos animais com cores mais vivas tiveram maior quantidade de acerto no aplicativo.

Em um segundo treinamento, foi realizado o aumento de dados para 2401 imagens e foi feito um ajuste fino do modelo com o descongelamento de camada posteriores da rede MobilenetV2, o intuito de utilizar essas camadas superiores pré treinadas junto com as camadas personalizadas, possibilita utilizar o prévio conhecimento da arquitetura da rede neural para contribuir no aumento da precisão do valor da acurácia do modelo. Após o novo treinamento atingiu um nível de acurácia de 90,22\%.

No segundo teste realizado diretamente no aplicativo, mostrou-se uma considerável melhora no desempenho em relação aos primeiros testes, obtendo um percentual de acerto de $88.41 \%$ nas imagens testadas, como é representado na tabela 2 . 


\begin{tabular}{|c|c|c|c|c|}
\hline Gênero/Espécie & $\begin{array}{c}\text { Total de imagens } \\
\text { testadas }\end{array}$ & $\begin{array}{c}\text { Número de } \\
\text { acertos }\end{array}$ & $\begin{array}{c}\text { Número de } \\
\text { erros }\end{array}$ & $\begin{array}{c}\text { Porcentagem média } \\
\text { de acerto }\end{array}$ \\
\hline Crotalus (Cascavel) & 20 & 16 & 4 & $80 \%$ \\
\hline $\begin{array}{c}\text { Bothrops (Jararaca) } \\
\text { Lachesis (Surucucu } \\
\text { pico de Jaca) }\end{array}$ & 36 & 31 & 5 & $86.11 \%$ \\
\hline $\begin{array}{c}\text { Micrurus (Coral) } \\
\text { Serpentes sem } \\
\text { Veneno }\end{array}$ & 32 & 32 & 4 & $88.88 \%$ \\
\hline Total & 16 & 36 & 4 & $93.75 \%$ \\
\hline
\end{tabular}

Tabela 2- análise da taxa de acerto do modelo no segundo teste.

Conclui-se que ao analisar a tabela 2 é notável a melhoria de desempenho e percentual de acerto em 3,66\% após utilizar o aumento de dados e aplicar no modelo as técnicas de Transfer learning utilizando e reaproveitando as camadas de uma rede pré treinada.

$\mathrm{Na}$ figura 6 são apresentadas as janelas principais do aplicativo. A primeira janela (A), ao abrir o aplicativo com dois botões, um para abrir a câmera do celular e tirar a foto do animal para fazer a classificação, e outro caso queira selecionar uma imagem da galeria do dispositivo.

A imagem (B) com a segunda tela mostra a imagem que foi selecionada e sua respectiva classificação com a porcentagem que contém a probabilidade da serpente pertencer a determinada espécie. $O$ aplicativo também possui uma tela onde são apresentadas as características e principais informações da espécie classificada.
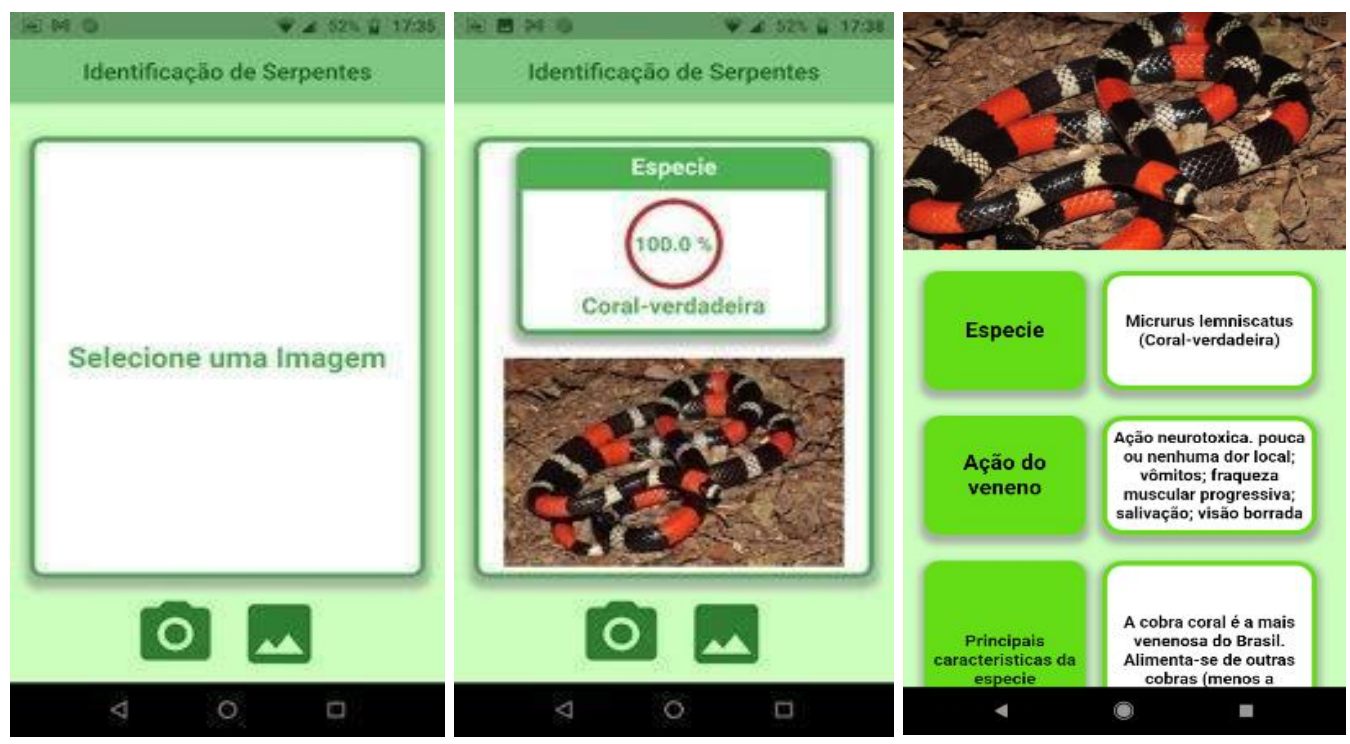


\section{Figura 6 - (A) Janela inicial. (B) Classificação da espécie. (C) Janela de informações.}

\section{Considerações finais}

O trabalho demonstra que a utilização dos métodos de Transfer Learning mostram-se totalmente eficazes quando possui um tamanho relativamente limitado de dados para treinamento do modelo de aprendizado profundo, além de proporcionar menor custo de implantação, assim como maior desempenho pois não requer um número grande de imagens e dispensa o treinamento de uma $\mathrm{CNN}$ começando do zero.

Com base nos resultados alcançados no aplicativo, obtendo uma taxa de acerto de $88.41 \%$ no teste com o aplicativo mobile e 90,22\% no nível de acurácia do modelo treinado, observa-se que esta ferramenta, pode ser um mecanismo viável para fazer o reconhecimento e identificação de espécies de serpentes peçonhentas no intuito de facilitar o atendimento a vítima de acidentes ofídicos.

Contudo, para um alcance ainda mais preciso nos resultados, é necessário um banco de imagens maior do que o que foi utilizado no trabalho para melhoria do conjunto de dados e diminuição da taxa de erro.

Para trabalhos futuros, almeja-se encorpar melhor o banco com mais imagens, para melhorar o desempenho durante o treinamento do modelo no sentido de aumentar seu nível de acurácia e por consequência, melhora da precisão do aplicativo.

\section{Referências}

Marques Junior, Luiz Carlos. Classificação de plantas daninhas em banco de imagens utilizando redes neurais convolucionais. Dissertação de mestrado. Universidade Estadual Paulista.2020.

Nardelli, Rudiard. Aplicativo de celular para identificação das principais espécies comerciais de camarões. Instituto Federal Catarinense.2020.

Britto, Larissa, Luciano Pacífico, Matheus Silva, and Teresa Ludermir. "Reconhecimento de Plantas Medicinais Usando Características de Cor, Textura e Forma." Anais do XVII Encontro Nacional de Inteligência Artificial e Computacional, Evento Online, 2020. SBC, 2020.

Osório, Fernando Santos, BITTENCOURT João Ricardo. Sistemas inteligentes baseados em Redes Neurais Artificiais aplicados ao Processamento de Imagens. I WORKSHOP DE INTELIGÊNCIA ARTIFICIAL UNISC - Universidade de Santa Cruz do Sul Departamento de Informática- Junho 2000.

Sandler, M., Howard, A., Zhu, M., Zhmoginov, A., and Chen, L.-C. (2018). MobileNetV2: Inverted residuals and linear bottlenecks. In IEEE Conference on Computer Vision and Pattern Recognition (CVPR), pages 4510-4520, Salt Lake City, Utah, Estados Unidos. IEEE Press.

Ginês, Daniel. ESTABILIS. 10 ferramentas / Frameworks de código aberto para IA. 2018. Disponível em: https://blog.estabil.is/10-ferramentas-frameworks-de-codigoaberto-para-ia/ Acesso em: 04 set de 2020. 
Devmedia. Devcast: o que é flutter? Disponivel em: https://www.devmedia.com.br/flutter/ Acesso em 04 de set. 2020.

Gama Academy. Como o aprendizado por transferência pode tornar o machine learning mais eficiente. Disponivel em: https://sitev1.gama.academy/blog/desenvolvimento/aprendizado-por-transferencia-machinelearning. Acesso em 04 de Set. 2020.

Tensorflow. Implante modelos de machine learning em dispositivos móveis e de internet das coisas (IoT na sigla em Inglês). Disponível em: https://www.tensorflow.org/lite?hl=pt-br. Acesso em: 04 de set. 2020.

Tensorflow. Transfer learning and fine-tuning. Disponivel em: https://www.tensorflow.org/tutorials/images/transfer_learning. Acesso em 04 de set. 2020.

Coros Saúde. O Brasil copatrocinou resolução para o problema dos acidentes ofídicos no mundo. 2018. Disponível em: https://www.saude.gov.br/noticias/svs/43413-brasilcopatrocinou-resolucao-para-o-problema-dos-acidentes-ofidicos-no-mundo. Acesso em: 04 de set. 2020.

Bezerra, Mirthyan. Serpente, escorpião e abelha: os animais peçonhentos que mais matam no país. UOL NOTÍCIAS. 2019. Disponível em: https://noticias.uol.com.br/saude/ultimas-noticias/redacao/2019/05/06/mortesanimais-peconhentos-brasil.htm. Acesso em:04 de set. 2020.

Ministério Da Saúde. Acidentes por animais peçonhentos: o que fazer e como evitar. Disponível em https://www.saude.gov.br/saude-de-a-z/acidentes-por-animaispeconhentos-serpentes. Acesso em: 04 de set. 2020.

Greenme. Atlas das Serpentes Brasileiras: o maior estudo sobre as espécies de serpentes no Brasil. 2020. Disponível em: https://www.greenme.com.br/informarse/animais/41063-maior-estudo-sobreserpentes-brasileiras-atlas/. Acesso em: 04 de set. 2020.

Ricmais. Veja o que fazer se encontrar animais silvestres. 2020. Disponível em: https://ricmais.com.br/noticias/animais-silvestres/. Acesso em: 04 de set. 2020. 